\title{
In-medium Properties of B and D Mesons
}

\author{
H. Sundu ${ }^{1, a}, K . A z i z i^{2, b}$, and N. Er ${ }^{3, c}$ \\ ${ }^{1}$ Department of Physics, Kocaeli University, 41380 Izmit, Turkey \\ ${ }^{2}$ Department of Physics, Doğuş University, Acıbadem-Kadıköy, 34722 İstanbul, Turkey \\ ${ }^{3}$ Department of Physics, Abant Izzet Baysal University, Gölköy Kampüsü, 14980 Bolu, Turkey
}

\begin{abstract}
The shifts in the masses and decay constants of B and D mesons in nuclear medium are calculated in the frame work of QCD sum rules. The results obtained are compared with the existing theoretical predictions.
\end{abstract}

\section{Introduction}

To better analyze the results of heavy ion collision experiments and understand the internal structures of the dense astrophysical objects like neutron stars, we need the study of the in-medium properties of hadrons. Moreover, it can be useful for understanding the non-perturbative dynamics as well as the vacuum structure of QCD. In the literature, both the experimental and theoretical studies on the properties of hadrons in-medium have received considerable attention [1-8]. In the present work, we calculate the shifts in the masses and decay constants of $B$ and $D$ mesons in nuclear medium in the framework of QCD sum rules.

\section{QCD sum rules for modifications of the masses and decay constants of the $D$ and $B$ mesons in nuclear medium}

In this section, we obtain QCD sum rules for the shifts in the masses and decay constants of $D$ and $B$ mesons in nuclear matter. We start with the following two-point correlation function:

$$
\Pi(q)=i \int d^{4} x e^{i q \cdot x}\left\langle\mathcal{T}\left[J_{B[D]}(x) J_{B[D]}^{\dagger}(0)\right]\right\rangle_{\rho_{N}}=\Pi_{0}(q)+\Pi_{N}(q) \simeq \Pi_{0}(q)+\frac{\rho_{N}}{2 M_{N}} T_{N}(q),
$$

where $\Pi_{0}(q)$ and $\Pi_{N}(q)$ are vacuum and the static one-nucleon parts in Fermi gas approximation for the nuclear matter, respectively. Here, $\mathcal{T}$ is the time ordering operator, $\rho_{N}$ is the density of the nuclear matter, $M_{N}$ is the mass of the nucleon and $J_{B[D]}(x)$ denotes the interpolating current of the $B[D]$ meson. In order to calculate the shifts in the values of the masses and decay constants, we consider the following forward scattering amplitude $T_{N}(q)$

$$
T_{N}\left(q_{0}=\omega, \mathbf{q}\right)=i \int d^{4} x e^{i q \cdot x}\left\langle N(p)\left|\mathcal{T}\left[J_{B[D]}(x) J_{B[D]}^{\dagger}(0)\right]\right| N(p)\right\rangle,
$$

\footnotetext{
a e-mail: hayriye.sundu@kocaeli.edu.tr

be-mail: kazizi@dogus.edu.tr

ce-mail: nuray@ibu.edu.tr
} 
where $q^{\mu}=(\omega, \mathbf{q})$ is the four-momentum of the meson and $|N(p)\rangle$ represents the isospin and spin averaged static nucleon state. The interpolating current of the pseudoscalar $B[D]$-meson can be written in terms of the quark fields as

$$
J_{B[D]}(x)=\frac{\bar{u}(x) i \gamma_{5} b[c](x)+\bar{b}[\bar{c}](x) i \gamma_{5} u(x)}{2} .
$$

where $u(x), b(x)$ and $c(x)$ are quark fields.

Following the general philosophy of the QCD sum rule method, the aforementioned correlation function can be calculated both in terms of the hadronic parameters called the physical or phenomenological side, and in terms of the QCD parameters called the theoretical or QCD side. These two representations are matched using dispersion relations to obtain QCD sum rules for the shifts in the masses and leptonic decay constants of the $B$ and $D$ mesons. Finally we apply Borel transformation to suppress the contribution of the higher states and continuum.

In the physical side, the forward scattering amplitude $T_{N}(\omega, \mathbf{q})$ is calculated in the limit $\mathbf{q} \rightarrow 0$, around $\omega=m_{B[D]}$. Near the pole position of the pseudoscalar meson, the $T_{N}(\omega, 0)$ is related to the T-matrix for the forward $B[D]-N$ scattering amplitude and can be written as the following dispersion integrals [11]:

$$
T_{N}(\omega, 0)=\int_{-\infty}^{+\infty} d u \frac{\rho(u, \mathbf{q}=0)}{u-\omega-i \varepsilon}=\int_{0}^{\infty} d u^{2} \frac{\rho(u, \mathbf{q}=0)}{u^{2}-\omega^{2}},
$$

where $\omega^{2} \neq$ positive real number and $\rho(u, \mathbf{q}=0)$ is the spin-averaged spectral density. After some straightforward calculations (see also [12]), the physical side of correlation function is obtained :

$$
\begin{aligned}
\Pi^{P H Y S}(\omega, 0) & \propto \frac{f_{B[D]}^{2} m_{B[D]}^{4}}{m_{b[c]}^{2}\left(m_{B[D]}^{2}-\omega^{2}\right)}+\frac{\rho_{N}}{2 M_{N}}\left\{\frac{a}{\left(m_{B[D]}^{2}-\omega^{2}\right)^{2}}+\frac{b}{m_{B[D]}^{2}-\omega^{2}}\right\} \\
& \simeq \frac{2 M_{N} f_{B[D]}^{2} m_{B[D]}^{4}+\rho_{N} m_{b[c]}^{2} b}{2 M_{N} m_{b[c]}^{2}\left[\left(m_{B[D]}^{2}-\frac{\rho_{N} m_{b[c]}^{2}}{2 M_{N} f_{B[D]}^{2} m_{B[D]}^{4}} a\right)-\omega^{2}\right]}
\end{aligned}
$$

where $f_{B[D]}$ is the leptonic decay constant of the $B[D]$-meson, $a$ and $b$ are the phenomenological parameters.

Using the modified mass in nuclear matter, $m_{B[D]}^{*}=m_{B[D]}+\delta m_{B[D]}=\sqrt{m_{B[D]}^{2}+\Delta m_{B[D]}^{2}}$, we obtain the shifts in the mass and leptonic decay constant of $B[D]$ meson as:

$$
\begin{aligned}
\delta m_{B[D]} & =2 \pi \frac{M_{N}+m_{B[D]}}{M_{N} m_{B[D]}} \rho_{N} a_{B[D]}, \\
\delta f_{B[D]} & =\frac{m_{b[c]}^{2}}{2 f_{B[D]} m_{B[D]}^{4}}\left(\frac{\rho_{N}}{2 M_{N}} b-\frac{4 f_{B[D]}^{2} m_{B[D]}^{3}}{m_{b[c]}^{2}} \delta m_{B[D]}\right),
\end{aligned}
$$

where the parameter $a_{B[D]}$ is the $B[D]-N$ scattering length [11]. In order to calculate the shifts in the mass and decay constant, we need to calculate the phenomenological parameters $a$ and $b$ using the forward scattering amplitude calculated both in hadronic and QCD sides.

In the low energy limit $\omega \rightarrow 0$, the $T_{N}^{H A D}(\omega, 0)$ is equivalent to the Born term $T_{N}^{B o r n}(\omega, 0)$. Hence, we can write the forward scattering amplitude in hadronic side as:

$$
T_{N}^{H A D}(\omega, 0)=T_{N}^{B o r n}(\omega, 0)+\frac{a}{\left(m_{B[D]}^{2}-\omega^{2}\right)^{2}}+\frac{b}{m_{B[D]}^{2}-\omega^{2}}+\frac{c}{s_{0}^{2}-\omega^{2}},
$$


with the condition

$$
\frac{a}{m_{B[D]}^{4}}+\frac{b}{m_{B[D]}^{2}}+\frac{c}{s_{0}}=0
$$

The Born term can be determined by the Born diagrams at the tree level $[9,11]$. To calculate it, we consider the contributions of the baryons $\Lambda_{b[c]}$ and $\Sigma_{b[c]}$ in the medium produced by the interaction of $\mathrm{B}[\mathrm{D}]$ with the nucleon, i.e.

$$
\begin{array}{ll}
B^{-}(b \bar{u})+p(u u d) \text { or } n(u d d) & \rightarrow \Lambda_{b}^{0}(u d b) \text { or } \Sigma_{b}^{-}(d d b) \\
D^{0}(c \bar{u})+p(u u d) \text { or } n(u d d) & \rightarrow \quad \Lambda_{c}^{+}, \Sigma_{c}^{+}(u d c) \text { or } \Sigma_{c}^{0}(d d c)
\end{array}
$$

We obtain the Born term $T_{N}^{B o r n}(\omega, 0)$ as [9]:

$$
T^{B o r n}(\omega, 0)=\frac{2 M_{N}\left(M_{N}+M_{\mathcal{B}}\right) m_{B[D]}^{4} f_{B[D]}^{2}}{\left[\omega^{2}-\left(M_{N}+M_{\mathcal{B}}\right)^{2}\right]\left(\omega^{2}-m_{B[D]}^{2}\right)^{2}\left(m_{u}+m_{b[c]}\right)^{2}} g_{N B[D] \mathcal{B}}^{2}\left(\omega^{2}\right) .
$$

where $\mathcal{B}$ denotes the $\Lambda_{b[c]}$ or $\Sigma_{b[c]}$ baryon and $g_{N B[D] \mathcal{B}}\left(\omega^{2}\right)$ is the strong coupling constant among the $B[D]$ meson, nucleon and $\mathcal{B}$ baryon.

The final form of the hadronic side of the current-nucleon forward scattering amplitude is obtained after double Borel transformation as (see also $[9,12]$ )

$$
\begin{aligned}
\widehat{\mathbf{B}} T_{N}^{P H Y S} & =a\left(\frac{1}{M^{2}} e^{-m_{B[D]}^{2} / M^{2}}-\frac{s_{0}}{m_{B[D]}^{4}} e^{-s_{0} / M^{2}}\right)+b\left(e^{-m_{B[D]}^{2} / M^{2}}-\frac{s_{0}}{m_{B[D]}^{2}} e^{-s_{0} / M^{2}}\right) \\
& +\frac{2 f_{B[D]}^{2} m_{B[D]}^{4} M_{N}\left(M_{N}+M_{\mathcal{B}}\right)}{\left[\left(M_{N}+M_{\mathcal{B}}\right)^{2}-m_{B[D]}^{2}\right]\left(m_{u}+m_{b[c]}\right)^{2}} g_{N B[D] \mathcal{B}}^{2} \\
& \times\left[-\frac{e^{-\left(M_{N}+M_{\mathcal{B}}\right)^{2} / M^{2}}}{\left(M_{N}+M_{\mathcal{B}}\right)^{2}-m_{B[D]}^{2}}+\left(\frac{1}{\left(M_{N}+M_{\mathcal{B}}\right)^{2}-m_{B[D]}^{2}}-\frac{1}{M^{2}}\right) e^{-m_{B[D]}^{2} / M^{2}}\right] .
\end{aligned}
$$

In the QCD side, we obtain the forward scattering amplitude by inserting the explicit form of the interpolating current $J_{B[D]}$ into Eq. (2) as:

$$
T_{N}^{O P E}=\frac{i}{4} \int d^{4} x e^{i q . x}\left\langle N(p)\left|\operatorname{Tr}\left[S_{Q}(-x) \gamma_{5} S_{u}(x) \gamma_{5}+S_{u}(-x) \gamma_{5} S_{Q}(x) \gamma_{5}\right]\right| N(p)\right\rangle
$$

where $S_{u}$ is light quark and $S_{Q}$ with $Q=b$ or $c$ is the heavy quark propagator. The next step is to use the expressions of the quark propagators and perform the trace and integrals. After lengthy calculations, we get the QCD side of the $T_{N}$ function in the rest frame of the nuclear matter in Borel 
scheme as:

$$
\begin{aligned}
\widehat{\mathbf{B}} T_{N}^{Q C D}= & \frac{1}{3} \frac{e^{-m_{Q}^{2} / M^{2}}}{M^{4}}\left\{-m_{Q}\left(-2 m_{Q}^{2}+M^{2}+2 p_{0}^{2}\right)\left\langle\bar{q} g_{s} \sigma G q\right\rangle_{N}\right. \\
& -4 m_{Q}\left(m_{Q}^{2}-2 M^{2}+4 p_{0}^{2}\right)\left\langle\bar{q} D_{0} D_{0} q\right\rangle_{N} \\
& +4 M^{2}\left(-m_{Q}^{2}+M^{2}+4 p_{0}^{2}\right)\left\langle q^{\dagger} i D_{0} q\right\rangle_{N} \\
& \left.+2 M^{2}\left[2 m_{Q}^{2} m_{u}-3 m_{Q} M^{2}+m_{u}\left(M^{2}-2 p_{0}^{2}\right)\right]\langle\bar{q} q\rangle_{N}\right\} \\
& +\frac{1}{12 \pi^{2}}\left\langle g_{s}^{2} G^{2}\right\rangle_{N} \int_{0}^{\infty} d \alpha \frac{e^{m_{Q}^{2} /\left(4 \alpha-M^{2}\right)} m_{Q}}{\left(4 \alpha-M^{2}\right)^{4}}\left\{16 \alpha^{2}\left(m_{Q}+3 m_{u}\right)\right. \\
& +M^{2}\left(-m_{Q}^{2} m_{u}+3 m_{Q} M^{2}+3 m_{u} M^{2}\right) \\
& \left.-4 \alpha\left(m_{Q}^{3}-m_{Q}^{2} m_{u}+4 m_{Q} M^{2}+6 m_{u} M^{2}\right)\right\} \theta\left[\frac{1}{-4 \alpha+M^{2}}\right] \\
& -\frac{m_{Q} e^{-m_{Q}^{2} / M^{2}}}{M^{2}}\left\langle\bar{q} g_{s} \sigma G q\right\rangle_{N} .
\end{aligned}
$$

where $M$ is the Borel mass parameter.

Finally, we equate the Borel transformed physical and QCD sides of the $\widehat{\mathbf{B}} T_{N}$ function to find QCD sum rules for the parameters $a$ and $b$. Since the functions $a$ and $b$ are very lengthy functions, we do not present their explicit expression here.

\section{Numerical results}

The sum rules for the parameters $a$ and $b$ contain two auxiliary parameters: the Borel parameter $M^{2}$ and the continuum threshold $s_{0}$. Since these are not physical parameters, the results of the parameters $a$ and $b$ should be practically independent of them. Therefore, we shall find their working regions such that these parameters weakly depend on these auxiliary parameters. Our numerical analysis show that in the intervals $25 \mathrm{GeV}^{2} \leq M^{2} \leq 40 \mathrm{GeV}^{2}$ and $4 \mathrm{GeV}^{2} \leq M^{2} \leq 8 \mathrm{GeV}^{2}$ respectively in the $B$ and $D$ channels, the dependence of the shifts in the physical quantities are weak. Also, we see that in the intervals $34 \mathrm{GeV}^{2} \leq s_{0} \leq 38 \mathrm{GeV}^{2}$ and $5.6 \mathrm{GeV}^{2} \leq s_{0} \leq 6.4 \mathrm{GeV}^{2}$ respectively for the $B$ and $D$ mesons, the results demonstrate weak dependence on the continuum threshold. To see how the results depend on the Borel mass parameter, we plot the dependence of the shift of the decay constant of the $B[D]$-meson under consideration versus $M^{2}$ for different values of the continuum threshold in figure 1. Making use of the working regions for auxiliary parameters and taking into account all systematic uncertainties, we obtain the numerical results of the shifts in mass and decay constant for $B[D]-$ meson as presented in table 1 . We also compare our results on the mass shifts with the existing theoretical predictions. Our result on the mass shift in $D$ channel is in a good consistency with the result of [9]. On the other hand, we see that our result in this channel is the same in magnitude with the prediction of [10], but with opposite sign. As far as the shift in the mass of $B$ channel is considered, our result is different in both sign and magnitude with the only existing prediction [10]. Our results on the leptonic decay constant shifts in $B$ and $D$ channels can be checked in future experiments.

In summary, we calculated the shifts in the masses and decay constants of the pseudoscalar $B$ and $D$ mesons in nuclear matter via the QCD sum rules. Our results obtained in the present work can help us not only analyze the future experimental data at different heavy ion collision experiments, but also 

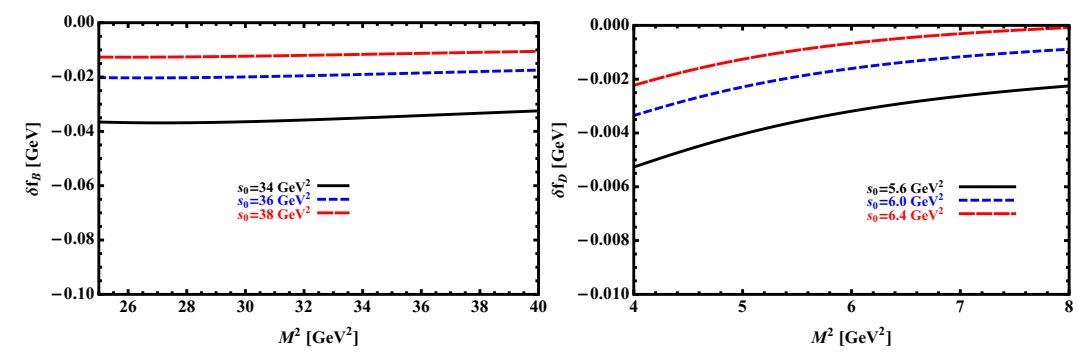

Figure 1. The shift of $B$ meson's decay constant in nuclear matter versus Borel mass $M^{2}$ at three different values of continuum threshold (left panel). The same, but for shift in decay constant of the $D$ meson (right panel).

Table 1. Average values of the shifts in the masses and decay constants of the $B$ and $D$ mesons.

\begin{tabular}{lllll}
\hline & $\delta m_{B}(\mathrm{GeV})$ & $\delta m_{D}(\mathrm{GeV})$ & $\delta f_{B}(\mathrm{GeV})$ & $\delta f_{D}(\mathrm{GeV})$ \\
\hline Present Work & $-0.242 \pm 0.062$ & $-0.046 \pm 0.007$ & $-0.023 \pm 0.007$ & $-0.002 \pm 0.001$ \\
\hline$[9]$ & - & $-0.048 \pm 0.008$ & - & - \\
\hline$[10]$ & $\sim 0.060$ & $\sim 0.045$ & - & - \\
\hline
\end{tabular}

better understand the perturbative and non-perturbative natures of QCD. The results obtained for the shifts in masses especially for those in the decay constants can also be used in theoretical calculations of the electromagnetic properties of the considered mesons as well as their strong couplings with other hadrons in nuclear medium.

\section{Acknowledgment}

This work has been supported in part by the Scientific and Technological Research Council of Turkey (TUBITAK) under the research project 114F018.

\section{References}

[1] Elisa Fioravanti, AIP Conf.Proc. 1432, 1-434 (2012); arXiv:1206.2214.

[2] B. Friman et al, "The CBM physics book: Compressed Baryonic Matter in Laboratory Experiments", Springer Heidelberg.

[3] http://www.gsi.de/fair/experiments/CBM/index e.html.

[4] http://www-panda.gsi.de/auto/phy/ home.htm.

[5] E. G. Drukarev and E. M. Levin, Pis'ma Zh. Eksp. Teor. Fiz. 48, 307 (1988).

[6] E. G. Drukarev and E. M. Levin, Nucl. Phys. A 511, 679, (1990); 516, 715(E) (1990).

[7] T. Hatsuda, H. Hogaasen, M. Prakash, Phys. Rev. Lett. 66, 2851 (1991).

[8] C. Adami, G. E. Brown, Z. Phys. A 340, 93 (1991).

[9] A. Hayashigaki, Phys. Lett. B 487, 96 (2000).

[10] T. Hilger, R. Thomas, B. Kämpfer, Phys. Rev. C 79, 025202 (2009).

[11] Y. Koike and A. Hayashigaki, Prog. Theo. Phys. 98, 631 (1997).

[12] K. Azizi, N. Er, H. Sundu, arXiv:1405.3058[hep-ph]. 
